\title{
Research Article \\ Effect of Atomic Number on Plasma Pinch Properties and Radiative Emissions
}

\author{
Walid Sahyouni ${ }^{1}$ and Alaa Nassif $\mathbb{D}^{2}$ \\ ${ }^{1}$ Department of Physics, Al-Baath University, Homs, Syria \\ ${ }^{2}$ Faculty of Engineering, Al-Wataniya Private University, Hama, Syria \\ Correspondence should be addressed to Alaa Nassif; alaa.nassif.85@hotmail.com
}

Received 3 December 2020; Revised 24 May 2021; Accepted 24 June 2021; Published 12 July 2021

Academic Editor: Theocharis Kosmas

Copyright (c) 2021 Walid Sahyouni and Alaa Nassif. This is an open access article distributed under the Creative Commons Attribution License, which permits unrestricted use, distribution, and reproduction in any medium, provided the original work is properly cited. The publication of this article was funded by SCOAP ${ }^{3}$.

\begin{abstract}
The aim of the research is to examine the dependence of plasma pinch properties and radiation emissions on the atomic number of the operating gas within the dense plasma focus device (NX2) when using hydrogen and argon gases. Simulation was performed with Lee's code on an NX2 dense plasma focus at a constant gas pressure value $\left(P_{0}=0.5\right.$ torr $)$. The results showed that the minimum radius of the plasma focus in the case of the hydrogen plasma pinch was $0.30 \mathrm{~cm}$ and in the case of the argon plasma pinch $0.17 \mathrm{~cm}$, and this affected the value of the radiation emission as it was $7.8 \times 10^{-6} \mathrm{~J}$ and $11 \mathrm{~J}$ for the hydrogen and argon pinch, respectively. The energy of the ion beam released by the breakdown of the plasma pinch was found as $E_{\mathrm{n}}=23.8 \mathrm{~J}$ in the state of hydrogen and $E_{\mathrm{n}}=105 \mathrm{~J}$ in the state of argon.
\end{abstract}

\section{Introduction}

Since the discovery of the phenomenon of dense plasma focus in the 1960s by the scientists Mather and Fillipov $[1,2]$ and their design of two types of dense plasma focus devices were known by their names, a large number of researches and studies have been conducted that have dealt with this unique phenomenon from various aspects such as plasma focus dynamics, particle emission, and X-ray emission. The study of the dynamics of the plasma focus covered the formation and acceleration of the plasma layer inside the device [3] and the factors affecting such as the sheath current [4], pinch current [5], length of the insulator used to separate the anode and the cathode, the type of gas used within the device $[6,7]$, the parameters of the capacitor bank [8] and electrode engineering, and ion beam properties produced in dense plasma focus devices using various gases [9]. Due to the collapse of the plasma pinch after a short period of time (several ns) of its formation and emitting ions and electrons in opposite directions, many studies have been conducted that dealt with the possibility of benefiting from the emitted particle beams such as lithography $[10,11]$ and short-lived radioisotope produc- tion [12-15] thin film deposition [16, 17]. The soft X-ray emission from dense plasma focus is according to two mechanisms: linear radiation and continuum radiation (recombination and Bremsstrahlung radiation) [18-20]. The hard X-rays are also produced by the collisions of electron beams resulting from the collapse of the plasma pinch with the anode [21-23].

Lee's code is one of the most important programs used to simulate the phenomenon of plasma focus because of its important features, as it couples electrical circuit parameters with the dynamics and thermodynamics of the plasma focus and radiation, enabling a realistic simulation of all gross focus properties. The basic model is described in [24, 25]. This code has been used in many plasma focus devices such as the United Nations University/International Centre for Theoretical Physics Plasma Focus Facility (UNU/ICTP PFF) NX2 device and NX1 device.

In the practical applications mentioned above, the plasma pinch is the source of ion beams, where the characteristics of these beams (number of ions, flux, and energy) are related to the specifications of the device used and also depend on the type of gas used, and this has been discussed in a previous study [9]. In this study, we studied the effect of the difference 
in atomic number on the narrowing of the pinch (the ratio of the radius of the pinch to the radius of the anode) by performing a series of numerical experiments using Lee's code according to the following method.

\section{Brief Description of the Methodology}

Two gases with diverging atomic number values were chosen (hydrogen 1 and argon 18). A large number of numerical experiments were carried out using Lee's code on a dense plasma focus device, and the following is a brief description of them.

2.1. NX2 Dense Plasma Focus Device Description. It is a compact plasma focus (CPF) device that works with a group of capacitors arranged in parallel to decrease the stray (static) inductance $L_{0}$ in the external circuit down to as low as 15 $\mathrm{nH}$, where a group of 12 capacitors are used. This device operates at up to $16 \mathrm{~Hz}$ repetition rate and produces a peak current of $400 \mathrm{kA}$ at $11.5 \mathrm{kV}$. Due to the higher frequency of the firing of NX2, the stainless steel electrode needs to be cooled by circulating water to avoid excessive heating [26].

2.2. Lee Code Description. The code couples the electrical circuit with plasma focus (PF) dynamics, thermodynamics, and radiation. It has been used in the design and interpretation of Mather-type PF experiments and as a complementary facility to provide diagnostic reference numbers in all gases. Information computed includes axial and radial dynamics, soft X-ray emission characteristics, and yield for various applications including microelectronic lithography and optimization of machines. Plasma focus neutron yield calculations, current and neutron yield limitations, deterioration of neutron scaling (neutron saturation), radiative collapse, speed-enhanced plasma focus, current-stepped plasma focus, and extraction of diagnostic and anomalous resistance data from current signals have been studied using the code, which also produces reference numbers for fluence, flux, and energy of deuteron beams and ion beams for all gases [27].

\section{Results and Discussion}

The total discharge current path is the most important indicator of the overall performance of the plasma focus. It operates (energy rendering) for all dynamic, thermodynamic, electrodynamic, and radiative emission processes in the different stages of the plasma focus evolution. The wave function of the total discharge current path contains information on all previous processes. The general shape of the current path is governed by the capacitor bank, tube, and operating parameters. Therefore, the first step when studying the plasma focus is to study the discharge current starting from the moment of discharging the capacitor bank. This is what we did, where we found the wave function of the discharge current when using the hydrogen and argon gases.

Lee code version RADPFV6.1b [28] was used to simulate an NX2 dense plasma focus device with the following parameters [29].

The basic device parameters were preserved, and the gas pressure value was fixed to the value $P_{0}=0.5$ torr at which the highest value of the total current $\left(I_{\text {peak }}\right)$ was achieved, assuming that at this value of current the largest possible amount of energy stored in the capacitor bank was transferred to the pinching phase.

The results were as follows.

3.1. Hydrogen Gas. The waveform of the discharge current was taken from the moment of closing the switch to the end of the capacitor bank discharge as shown in Figure 1 where the highest value of the discharge current is $I_{\text {peak }}=$ $199 \mathrm{kA}$.

According to Lee's code and the waveform of the discharge current, the radial phase starts at $T_{\text {radial } / \text { start }}=0.377 \mu$ $\mathrm{s}$ and ends at $T_{\text {radial/end }}=0.446 \mu \mathrm{s}$, meaning that it lasts about $T_{\text {radial }}=0.069 \mathrm{~ns}$ and that the hydrogen plasma pinch time $T_{\text {pinch }}=9.7 \mathrm{~ns}$ and that the number of ions within the pinch is $n_{\mathrm{i}}=0.4 \times 10^{23} \mathrm{~m}^{-3}$ and the maximum temperature is $T_{\text {max }}=21.73 \times 10^{6} \mathrm{~K}$.

The simulation showed that the minimum pinch radius is $r_{\text {min }}=0.30 \mathrm{~cm}$ and the ratio of the pinch plasma radius to the anode radius is $r_{\min } / a=0.158$.

3.2. Argon Gas. By following the same procedure as in the case of hydrogen gas, the waveform of the discharge current is shown in Figure 2.

The highest value of the discharge current is $I_{\text {peak }}=362$ $\mathrm{kA}$.

The radial phase begins at $T_{\mathrm{radial} / \mathrm{start}}=0.829 \mu \mathrm{s}$ and ends at $T_{\text {radial/end }}=0.974 \mu \mathrm{s}$, that is, it lasts about $T_{\text {radial }}=0.144 \mu \mathrm{s}$ and the hydrogen plasma pinch time $T_{\text {pinch }}=17.3 \mathrm{~ns}$ and the number of ions within the pinch is $n_{\mathrm{i}}=1.9 \times 10^{23} \mathrm{~m}^{-3}$ and the maximum temperature is $T_{\max }=4.49 \times 10^{6} \mathrm{~K}$. We observed that the minimum pinch radius is $r_{\min }=0.17 \mathrm{~cm}$ and the ratio of the pinch plasma radius to the anode radius is $r_{\min } / a=0.091$.

By making a comparison between the value of the minimum pinch radius in the case of hydrogen, $r_{\min }=0.30 \mathrm{~cm}$, and argon, $r_{\min }=0.17 \mathrm{~cm}$, we note the lower value of $r_{\min }$ in the case of argon, which can be explained by the energy gain/loss in the case of argon plasma during the compression phase, and from Lee's code, we found the values of energy gain (Joule heating) and energy loss (radiation) shown in Table 1.

It is clear from Table 2 that the energy loss due to the radiation in the hydrogen plasma is very small, and thus, it is not a reason to reduce the plasma pinch radius.

From the results of the numerical simulation, we note that both the pinching time and the ratio of pinch plasma radius to the anode radius $\left(r_{\min } / a\right)$ in the case of argon gas were greater than those in hydrogen gas and thus a greater number of ions are trapped within the pinch $\left(\operatorname{argon} n_{\mathrm{i}}=1.9\right.$ $\times 10^{23} \mathrm{~m}^{-3}>$ hydrogen $\left.n_{\mathrm{i}}=0.4 \times 10^{23} \mathrm{~m}^{-3}\right)$; this increases the energy of the resulting ion beam. The simulation showed that the energy of the hydrogen ion beam released upon breakdown of the formed plasma pinch was $E_{\mathrm{n}}=23.8 \mathrm{~J}$, which constituted $0.9 \%$ of the operating energy, while in the case of argon $E_{\mathrm{n}}=105 \mathrm{~J}$ and $3.8 \%$ of the operating energy. 


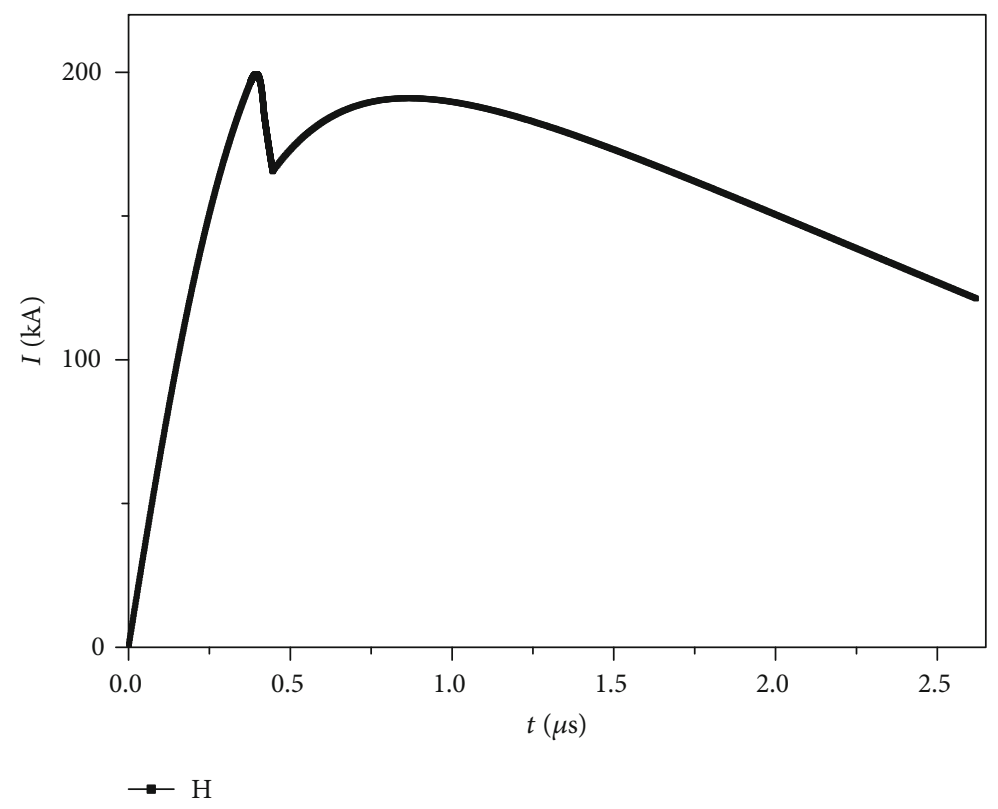

FIGURE 1: The waveform of the discharge current when hydrogen gas was used.

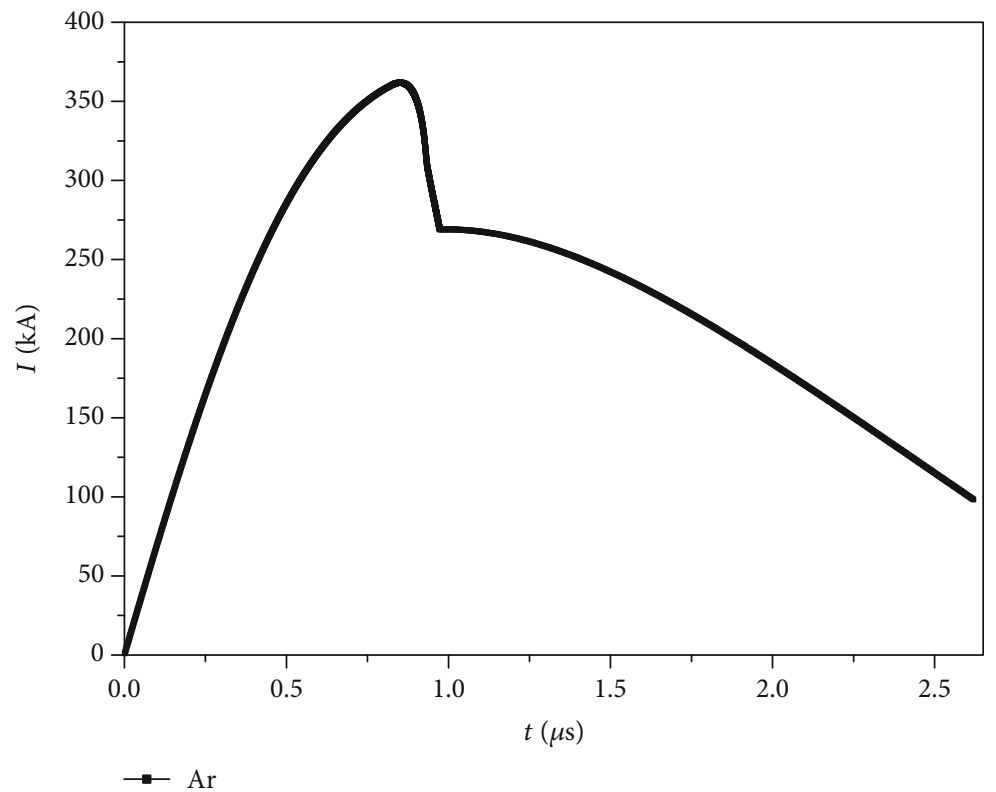

FIgURE 2: The waveform of the discharge current when argon gas was used.

TABle 1: NX2 dense plasma focus device parameters.

\begin{tabular}{|c|c|c|c|c|}
\hline Bank parameters & $\begin{array}{c}\text { Static inductance } \\
L_{0}=20 \mathrm{nH}\end{array}$ & $\begin{array}{c}\text { Capacity } \\
C_{0}=28 \mu \mathrm{F}\end{array}$ & $\begin{array}{c}\text { Stray resistance } \\
r_{0}=2.2 \mathrm{~m} \Omega\end{array}$ & \\
\hline Tube parameters & $\begin{array}{c}\text { Cathode radius } \\
b=4.1 \mathrm{~cm}\end{array}$ & $\begin{array}{c}\text { Anode radius } \\
a=1.9 \mathrm{~cm}\end{array}$ & $\begin{array}{c}\text { Anode length } \\
z_{0}=5 \mathrm{~cm}\end{array}$ & \\
\hline Operation parameters & $\begin{array}{c}\text { Voltage } \\
V_{0}=14 \mathrm{kV}\end{array}$ & $\begin{array}{c}\text { Pressure } \\
\mathrm{P}_{0}=0.5 \text { torr }\end{array}$ & & \\
\hline Model parameters & $f_{\mathrm{m}}=0.08$ & $f_{\mathrm{c}}=0.7$ & $f_{\mathrm{mr}}=0.2$ & $f_{\mathrm{cr}}=0.7$ \\
\hline
\end{tabular}


TABLe 2: Values of energy gain (Joule heating) and energy loss (radiation) in hydrogen and argon plasma.

\begin{tabular}{lcc}
\hline & $Q_{-}\{$Joule $\}$(in J) & $Q_{-}\{$radiation $\}$(in J) \\
\hline Hydrogen plasma pinch & $+4.85 \times 10^{\{-3\}}$ & $-7.8 \times 10^{\{-6\}}$ \\
Argon plasma pinch & +2.8 & -11.0 \\
\hline
\end{tabular}

By comparing these results with the results of the research [9], the energy of the ion beam was changed due to two factors, both the pinch's voltage and the number of effective charge, while in our study we focused on the effect of constriction of the pinch on the energy of ion beam as it was found that the lower constriction value (argon) increased the value of the ion beam energy because a greater number of ions are trapped inside the pinch.

\section{Conclusions}

This research evidenced the importance of the value of the atomic number of gas used in the dense plasma focus device on the formation of the plasma pinch during the compression phase. The study showed that in the case of the gas with a high atomic number (argon) the pinch narrowing was $r_{\min } / \mathrm{a}=0.091$ while in the case of the gas with a low atomic number (hydrogen) it was $r_{\min } / \mathrm{a}=0.158$, and thus, in the case of the argon plasma there was a greater transfer of energy to the plasma, thus increasing the radiation and as a result the energy value of the ion beam, resulting from the breakdown of the pinch increased later, as the energy of the argon ion beam was $E_{\mathrm{n}}=105 \mathrm{~J}$ while the energy of the hydrogen ion beam was $E_{\mathrm{n}}=23.8 \mathrm{~J}$.

\section{Data Availability}

Data are available upon authors' request; contact alaa. nassif.85@hotmail.com.

\section{Conflicts of Interest}

The authors declare that they have no conflicts of interest.

\section{References}

[1] J. W. Mather, "Formation of a high-density deuterium plasma focus," The Physics of Fluids, vol. 8, no. 2, pp. 366-377, 1965.

[2] N. V. Fillipov, T. I. Fillipova, and V. P. Vinogradov, "Dense high-temperature plasma in a non-cylindrical $Z$-pinch compression,” Nuclear Fusion, Supplement 2, pp. 577-587, 1962.

[3] A. Shakya, P. Gautam, and R. Khanal, "Comparison of plasma dynamics in plasma focus devices PF1000 and PF400," Journal of Nepal Physical Society, vol. 3, no. 1, pp. 55-59, 2016.

[4] M. S. Rafique, P. Lee, A. Patran, R. S. Rawat, and S. Lee, "Radiation emission correlated with the evolution of current sheath from a deuterium plasma focus," Journal of Fusion Energy, vol. 29, no. 3, pp. 295-304, 2010.

[5] S. Lee and S. H. Saw, "Pinch current limitation effect in plasma focus," Applied Physics Letters, vol. 92, no. 2, article 021503, 2008.
[6] S. Al-Hawat, M. Akel, S. Lee, and S. H. Saw, "Model parameters versus gas pressure in two different plasma focus devices operated in argon and neon," Journal of Fusion Energy, vol. 31, no. 1, pp. 13-20, 2012.

[7] S. Lee and S. H. Saw, "Plasma focus ion beam fluence and flux-for various gases," Physics of Plasmas, vol. 20, no. 6, article 062702, 2013.

[8] S. H. Saw, S. Lee, F. Roy et al., "In situdetermination of the static inductance and resistance of a plasma focus capacitor bank," Review of Scientific Instruments, vol. 81, no. 5, article 053505, 2010.

[9] S. Walid and N. Alaa, "Ions beam properties produced by NX2 plasma focus device with helium and nitrogen gas," American Journal of Modern Physics, vol. 8, no. 1, article 6221273, pp. 14, 2019.

[10] E. P. Bogolyubov, V. D. Bochkov, V. A. Veretennikov et al., "A powerful soft X-ray source for X-ray lithography based on plasma focusing," Physica Scripta, vol. 57, no. 4, pp. 488-494, 1998.

[11] Sing Lee, P. Lee, Guixin Zhang et al., "High rep rate high performance plasma focus as a powerful radiation source," IEEE Transactions on Plasma Science, vol. 26, no. 4, pp. 11191126, 1998.

[12] E. Angeli, A. Tartari, M. Frignani et al., "Production of radioisotopes within a plasma focus device," Nuclear Technology and Radiation Protection, vol. 20, no. 1, pp. 33-37, 2005.

[13] A. Talaei, S. M. Sadat Kiai, and A. A. Zaeem, "Effects of admixture gas on the production of ${ }^{18} \mathrm{~F}$ radioisotope in plasma focus devices," Applied Radiation and Isotopes, vol. 68, no. 12, pp. 2218-2222, 2010.

[14] M. V. Roshan, S. V. Springham, R. S. Rawat, and P. Lee, "Short-lived PET radioisotope production in a small plasma focus device," IEEE Transactions on Plasma Science, vol. 38, no. 12, pp. 3393-3397, 2010.

[15] M. Akel, S. Alsheikh Salo, S. Ismael, S. H. Saw, and S. Lee, "Interaction of the high energy deuterons with the graphite target in the plasma focus devices based on Lee model," Physics of Plasmas, vol. 21, no. 7, article 072507, 2014.

[16] O. Mangla, S. Roy, and K. K. Ostrikov, "Dense plasma focusbased nanofabrication of III-V semiconductors: unique features and recent advances," Nanomaterials, vol. 6, no. 1, p. 4, 2015.

[17] Z. Y. Pan, R. S. Rawat, M. V. Roshan et al., "Nanostructured magnetic CoPt thin films synthesis using dense plasma focus device operating at sub-kilojoule range," Journal of Physics D: Applied Physics, vol. 42, no. 17, article 175001, 2009.

[18] M. Akel and S. Lee, "Dependence of plasma focus argon soft Xray yield on storage energy, total and pinch currents," Journal of Fusion Energy, vol. 31, no. 2, pp. 143-150, 2012.

[19] Sing Lee, S. H. Saw, R. S. Rawat et al., "Correlation of measured soft X-ray pulses with modeled dynamics of the plasma focus," IEEE Transactions on Plasma Science, vol. 39, no. 11, pp. 31963202, 2011.

[20] M. Akel, S. Al-Hawat, and S. Lee, "Numerical experiments on soft X-ray emission optimization of nitrogen plasma in $3 \mathrm{~kJ}$ plasma focus SY-1 using modified lee model," Journal of Fusion Energy, vol. 28, no. 4, pp. 355-363, 2009.

[21] M. Mahtab, M. Taghipour, G. H. Roshani, and M. Habibi, "Approach to the highest HXR yield in plasma focus device using adaptive neurofuzzy inference system to optimize anode 
configuration," Journal of Experimental Physics, vol. 2014, Article ID 307403, 8 pages, 2014.

[22] F. Di Lorenzo, V. Raspa, P. Knoblauch, A. Lazarte, C. Moreno, and A. Clausse, "Hard X-ray source for flash radiography based on a $2.5 \mathrm{~kJ}$ plasma focus," Journal of Applied Physics, vol. 102, no. 3, article 033304, 2007.

[23] E. Fogliatto, J. González, M. Barbaglia, and A. Clausse, "A model of hard X-rays emission from free expanding plasmafocus discharges," Journal of Physics Conference Series, vol. 511, article 012036, 2014.

[24] S. Lee, "Plasma focus model yielding trajectory and structure. In radiation in plasmas. V. 2. Reviews from 1983 college on plasma physics," 1984, https://inis.iaea.org/.

[25] W. Sahyouni and A. Nassif, "Neon soft X-ray yield optimization from NX2 dense plasma focus device," Jordan Journal of Physics, vol. 11, no. 3, pp. 167-172, 2018.

[26] S. Lee and S. H. Saw, "Generation of soft X-ray (SXR) from plasma focus," International journal of engineering and technology, vol. 1, no. 1, p. 2010, 2010.

[27] S. Lee, "Plasma focus radiative model: review of the Lee model code," Journal of Fusion Energy, vol. 33, no. 4, pp. 319-335, 2014.

[28] S. Lee, "Radiative dense plasma focus computation package: RADPF," 2010, http://www.plasmafocus.net/.

[29] S. Lee and S. H. Saw, Scaling of Ion Beams from Plasma Focus in Various Gases, vol. 14, INTI International University Nilai Malaysia, 2013. 